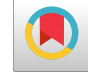

\title{
Vitamin D Status in Children with Nephrotic Syndrome
}

\author{
Parsa Yousefichaijan ${ }^{1}$, Aziz Eghbali ${ }^{2}$, Ali Khosrobeigi ${ }^{3,{ }^{*}}$, Hasan Taherahmadi ${ }^{4}$, Mohammad Rafiei $^{5}$, \\ Sima Tayebi ${ }^{2}$ and Ali Arjmand ${ }^{4}$ \\ ${ }^{1}$ Amirkabir Hospital, Department of Pediatric Nephrology, School of Medicine, Arak University of Medical Sciences, Arak, IR Iran \\ ${ }^{2}$ Khansary Hospital, Department of Oncology and Hematology, School of Medicine, Arak University of Medical Sciences, Arak, IR Iran \\ ${ }^{3}$ Department of Student Research Committee, School of Medicine, Arak University of Medical Sciences, Arak, IR Iran \\ ${ }^{4}$ Amirkabir Hospital, Department of Pediatrics, School of Medicine, Arak University of Medical Sciences, Arak, IR Iran \\ ${ }^{5}$ Department of Biostatistics, School of Medicine, Arak University of Medical Sciences, Arak, IR Iran \\ "Corresponding author: Ali Khosrobeigi, Students Research Committee of Arak University of Medical Sciences, Arak, IR Iran. Tel: +98-9188616847, E-mail: \\ ali.khosrobeigi@arakmu.ac.ir
}

Received 2017 May 01; Revised 2017 September 30; Accepted 2017 October 20.

\begin{abstract}
Background: Children with nephrotic syndrome (NS) are treated for 12 weeks with prednisolone regarding the damage to the bones. Deficiency in the level of vitamin D damages the bones. Assessment of vitamin D status in children with NS is based on serum 25OHD3 level taking into consideration the type of steroid susceptible (SS), steroid dependent (SD), and steroid resistance (SR) nephrotic syndrome (NS).

Methods: This study was a prospective study on children with the first visit of NS before treatment with prednisolone. The glomerular filtration rate (GFR) was calculated with Schwartz formula, and only sick people with normal GFR were included in the study. Analysis of 218 children aged 1-13 years, median 9.5 [108 with SSNS, 64 with SDNS, and 46 with SRNS] was performed. Serum concentrations of 1,25(OH)2 D3, calcium, phosphorus, and creatinine were measured. The correlation of 25OHD3 with the type of nephrotic syndrome, seasons, gender, and age was investigated.

Results: A total of 218 children were examined. Vitamin D level was deficient ( $<10 \mathrm{ng} / \mathrm{mL}$ ) in $79 \%$ of SRNS, $83 \%$ of SDNS, and $17 \%$ of SSNS group (P value 0.0001), insufficient (10 - $30 \mathrm{ng} / \mathrm{mL}$ ) in 81\% of SRNS, 73\% of SDNS, and 9\% of SSNS group (P value $=0.0003)$, and sufficient (30-150 ng/mL) in 91\% of SSNS, 17\% of SDNS, and 7\% of SRNS group (P value $=0.002$ ).

Conclusions: Our results suggest the necessity of developing new strategies in vitamin D supplementation in children with INS.
\end{abstract}

Keywords: Vitamin D Level, Nephrotic Syndrome, Prognostic Biomarker, Steroid Resistance

\section{Background}

About $90 \%$ of children with nephrotic syndrome are in the idiopathic nephrotic syndrome group. The idiopathic nephrotic syndrome is accompanied by primary glomerular disease $(1,2)$. These children show $3+$ or $4+$ proteinuria, and hematuria in microscopic level is shown in $20 \%$ of children. Protein excretion in urine exceeds $40 \mathrm{mg} / \mathrm{m}^{2} / \mathrm{h}$ (3). The creatinine value in serum is not significantly different between those with the steroid-sensitive nephrotic syndrome and those with nephrotic syndromes other than steroid-sensitive nephrotic syndrome (4). The level of albumin in serum is $<2.5 \mathrm{~g} / \mathrm{dL}$, cholesterol and triglyceride levels in serum are increased, and levels of serum complement are normal $(5,6)$. Approximately $80-90 \%$ of children respond to steroid therapy in three weeks. It has been documented that both an elevated steroids dose and a long time therapy are important factors in reducing relapse risk (7). Children with nephrotic syndrome should go to school and take part in physical activities (8).

Few foods contain vitamin D; the major natural source of vitamin D is synthesis in the skin. Vitamin D is built in the skin from cholesterol and its production depends on sun exposure (9).

Vitamin D, whether built in the skin or found in the diet, is inactive biologically; activation of vitamin D needs enzymatic conversion in the kidneys and liver (10).

Vitamin D has an important role in the metabolism and homeostasis of calcium. It is used to inhibit osteomalacia or rickets. In the general population, vitamin D supplementation is used for other health effects (11). Cholecalciferol is converted to calcidiol in the liver, and a part of calcidiol is converted to calcitriol in the kidneys (1).

\section{Methods}

This study was a prospective study conducted on children with the first visit of NS before treatment with prednisolone. Duration of this study was 2 years and 218 children were enrolled in the study. The glomerular filtration rate(GFR) was calculated using Schwartz formula, and only 
sick people with normal GFR were included in the study. Analysis of 218 children aged 1 - 13 years, the median of 9.5 [108 with SSNS, 64 with SDNS, and 46 with SRNS] was performed. Serum concentrations of $1,25(\mathrm{OH}) 2$ D3, calcium, phosphorus, and creatinine were measured. The correlation of 25OHD3 with the type of nephrotic syndrome, seasons, gender, and age was investigated.

\section{Results}

A total of 218 children were examined. Vitamin D level was deficient ( $<10 \mathrm{ng} / \mathrm{mL}$ ) in $79 \%$ of SRNS, $83 \%$ of SDNS, and $17 \%$ of SSNS group (P value $=0.0001)$, insufficient $(10$ - $30 \mathrm{ng} / \mathrm{mL}$ ) in $81 \%$ of SRNS, 73\% of SDNS, and 9\% of SSNS group $(P$ value $=0.0003)$, and sufficient $(30-150 \mathrm{ng} / \mathrm{mL})$ in 91\% of SSNS, $17 \%$ of SDNS, and 7\% of SRNS group (P value $=$ 0.002 ). Potential intoxication ( $>150 \mathrm{ng} / \mathrm{mL}$ ) was in $0 \%$ of NS. Vitamin D was observed in the study children with INS. There were no significant differences in serum calcium, phosphorus, and calcium x phosphorus product depending on the type of INS and gender. We compared vitamin $D$ level in patients with normal ranges so we did not need any control group.

\section{Discussion}

In our study, vitamin D level was lower in patients with SDNS and SRNS than in patients with SSNS; therefore, vitamin D level can be used as an indicator in the prognosis of NS in patients. Nielsen found that vitamin D in $93 \%$ of children with nephrotic syndrome was insufficient. They suggested that routine measurement of vitamin D status in children with Nephrotic syndrome at the diagnosis time could be a strategy for treating individuals if suffering from vitamin D deficiency. More studies are required to determine the role of such treatment (11). Banerjee et al. found that in sick people with Nephrotic Syndrome, store of vitamin D remained low for 12 weeks after Nephrotic Syndrome relapse while they found a rise in control levels with longer remission durations. Store of Vitamin D was not influenced by illness characteristics or therapy. More studies are needed to confirm these findings and evaluate the role of vitamin D in bones, particularly in frequent relapses (1). Further studies are important to demonstrate if treatment for vitamin D deficiency affects the bone mineral density and reduces the amount of albumin in the urine of children with NS.

\subsection{Conclusions}

Based on our results and the literature, we suggest that children may benefit from routine measurement of their vitamin D status at the time of diagnosis of NS for the first time or at relapse, so an individual strategy for treatment with vitamin $\mathrm{D}$ can be given in order to avoid the potential damages due to vitamin $\mathrm{D}$ deficiency.

\section{Acknowledgments}

This work was performed in partial fulfillment of the requirements for (doctor of medicine) of (Sima Tayebi), in School of medicine, Arak University of Medical Sciences, Arak, Iran.

\section{References}

1. Banerjee S, Basu S, Sengupta J. Vitamin D in nephrotic syndrome remission: a case-control study. Pediatr Nephrol. 2013;28(10):1983-9. doi: 10.1007/s00467-013-2511-y. [PubMed: 23708762].

2. Gulati S, Godbole M, Singh U, Gulati K, Srivastava A. Are children with idiopathic nephrotic syndrome at risk for metabolic bone disease? Am J Kidney Dis. 2003;41(6):1163-9. [PubMed:12776267].

3. Misra M, Pacaud D, Petryk A, Collett-Solberg PF, Kappy M; Drug, et al. Vitamin D deficiency in children and its management: review of current knowledge and recommendations. Pediatrics. 2008;122(2):398417. doi: 10.1542/peds.2007-1894. [PubMed: 18676559].

4. Odaka J, Kanai T, Ito T, Saito T, Aoyagi J, Betsui H, et al. Apolipoprotein C-I Levels Are Associated with the Urinary Protein/Urinary Creatinine Ratio in Pediatric Idiopathic Steroid-Sensitive Nephrotic Syndrome: A Case Control Study. Int J Nephrol. 2017;2017:6392843. doi: 10.1155/2017/6392843. [PubMed: 28250989].

5. Huang JP, Bai KM, Wang BL. Vitamin D and calcium metabolism in children with nephrotic syndrome of normal renal function. Chin Med J (Engl).1992;105(10):828-32. [PubMed: 1291200].

6. Grymonprez A, Proesmans W, Van Dyck M, Jans I, Goos G, Bouillon R. Vitamin D metabolites in childhood nephrotic syndrome. Pediatr Nephrol.1995;9(3):278-81. [PubMed: 7632510].

7. Yousefichaijan P, Rezagholizamenjany M, Rafiei F, Taherahmadi $\mathrm{H}$, Rafiei M. The Relationship between Blood Biomarkers Level and the Prognosis of Nephrotic Syndrome in the Children. Int J Pediatr. 2016;4(9):3489-97.

8. Beck-Nielsen SS, Jensen TK, Gram J, Brixen K, Brock-Jacobsen B. Nutritional rickets in Denmark: a retrospective review of children's medical records from 1985 to 2005. Eur J Pediatr. 2009;168(8):941-9. doi: 10.1007/s00431-008-0864-1. [PubMed:18985384].

9. Molina P, Gorriz JL, Molina MD, Peris A, Beltran S, Kanter J, et al. The effect of cholecalciferol for lowering albuminuria in chronic kidney disease: a prospective controlled study. Nephrol Dial Transplant. 2014;29(1):97-109. doi: 10.1093/ndt/gft360. [PubMed: 23975842].

10. Zaniew M, Jarmolinski T. Vitamin D status and bone density in steroid-treated children with glomerulopathies: effect of cholecalciferol and calcium supplementation. Adv Med Sci. 2012;57(1):88-93. doi: 10.2478/v10039-012-0016-8. [PubMed: 22472471].

11. Nielsen CA, Jensen JE, Cortes D. Vitamin D status is insufficient in the majority of children at diagnosis of nephrotic syndrome. Dan Med J. 2015;62(2). [PubMed: 25634505]. 